\title{
Vertex operators for production of baryon resonances in nucleon-gamma collisions with preserving gauge invariance and analyticity
}

\author{
A.V. Anisovich, V.V. Anisovich, A.V. Sarantsev, \\ National Research Centre "Kurchatov Institute": Petersburg Nuclear Physics \\ Institute, Gatchina, 188300, Russia \\ Vertex operators for photo- and electro-production of baryon states with arbitrary \\ spin-parity, $\gamma+N \rightarrow B\left(J^{P}\right)$, are constructed. The operators obey gauge invariance and \\ analyticity constraints. Analyticity is realized as a requirement of the generalized Siegert \\ theorem for vertex form factors. \\ PACS numbers: 13.60.Le, 11.55.Jy., 12.40.Yx
}

\section{Introduction}

The photo- and electro-production of mesons off nucleons provide a direct way for the identification of baryon resonances, that was at the times of the discovery of low-lying states [1.7] as well as at recent studies [8 16].

The present work is devoted to the baryon-nucleon-gamma vertex, $B_{J^{P}}(p) \rightarrow$ $N_{\frac{1}{2}}(k)+\gamma_{1^{-}}(q)$, where $p, k, q$ are correspondingly four-momenta of baryon state with spin-parity $J^{P}$, nucleon and gamma; energy-momentum conservation reads $p=k+q$. Our consideration is based on results of ref. [17] where the case of real photon $\left(q^{2}=0\right)$ was considered for any $J^{P}$. An advantige of the used classification of operators is the fixation not only spin-parity but helicity amplitudes as well that is convenient in data fit procedure.

The case of arbitrary $q^{2}$ is the subject of this paper. A problem in the construction of the gauge invariant spin-momentum operators appears because of the pole singularity $\left(1 / q^{2}\right)$ inherent to the spin part of a vector particle propagator. We eliminate the singularity by adding the longitudinal spin operator, which is nilpotent at $q^{2}=0$, as a cancellation term. Nilpotent operators for improving the analytical stucture of operators were used for meson states in [18]20] (see also [21 22]). The new operators impose a requirement for the decay form factors which is known for real photons as the Siegert theorem [23].

The present consideration is based on spin-operator expansion technique presented in detail in 21]22], necessary elements of this technique are given in Appendices $\mathrm{A}, \mathrm{B}$. 


\section{Vertices and operators}

According to the classification used in [17] we have two types of vertices:

(i) the (+)-vertices for transitions $B\left(J^{P}\right) \rightarrow N\left(\frac{1}{2}^{+}\right) \gamma$ with spin-parity of the decaying states equal to $J^{P}=\frac{1}{2}^{-}, \frac{3}{2}^{+}, \frac{5}{2}^{-}, \frac{7}{2}^{+}, \ldots$ and

(ii) the (-)-vertices for decaying states with $J^{P}=\frac{1}{2}^{+}, \frac{3}{2}^{-}, \frac{5}{2}^{+}, \frac{7}{2}^{-}, \ldots$

\subsubsection{The (+)-vertices}

Vertices of the $(+)$-states $\left(J^{P}=\frac{1}{2}^{-}, \frac{3}{2}^{+}, \frac{5}{2}^{-}, \ldots\right)$ read:

$$
\begin{aligned}
& G_{\alpha_{1} \ldots \alpha_{n}}^{(a+)}\left(k_{\perp}\right)=G_{n+\frac{1}{2}}^{(a+)}\left(s, q^{2}\right) V_{\alpha_{1} \ldots \alpha_{n}}^{(a+) \mu}\left(k_{\perp}\right) \epsilon_{\mu}^{\perp q}, \quad a=1,2,3, \\
& V_{\alpha_{1} \ldots \alpha_{n}}^{(1+) \mu}\left(k_{\perp}\right)=i \gamma_{\mu}^{\perp p} \gamma_{5} X_{\alpha_{1} \ldots \alpha_{n}}^{(n)}\left(k_{\perp}\right), \\
& V_{\alpha_{1} \ldots \alpha_{n}}^{(2+) \mu}\left(k_{\perp}\right)=i \gamma_{\nu}^{\perp p} \gamma_{5} X_{\mu \nu \alpha_{1} \ldots \alpha_{n}}^{(n+2)}\left(k_{\perp}\right), \\
& V_{\alpha_{1} \ldots \alpha_{n}}^{(3+) \mu}\left(k_{\perp}\right)=i \gamma_{\nu}^{\perp p} \gamma_{5} X_{\nu \alpha_{1} \ldots \alpha_{n-1}}^{(n)}\left(k_{\perp}\right) g_{\alpha_{n} \mu}^{\perp p} .
\end{aligned}
$$

Here $n=J-\frac{1}{2}$ and

$$
\begin{aligned}
& s=p^{2}=(k+q)^{2}, \quad(q p)=\frac{1}{2}\left(p^{2}-M_{N}^{2}+q^{2}\right) \\
& k=k_{\perp}+p \frac{(k p)}{p^{2}}, \quad q=-k_{\perp}+p \frac{(q p)}{p^{2}}, \\
& \gamma_{\mu}^{\perp p}=\gamma_{\mu^{\prime}} g_{\mu^{\prime} \mu}^{\perp p}=\gamma_{\mu^{\prime}}\left(g_{\mu^{\prime} \mu}-\frac{p_{\mu^{\prime}} p_{\mu}}{p^{2}}\right) .
\end{aligned}
$$

The form factors $G_{n+\frac{1}{2}}^{(a+)}\left(s, q^{2}\right)$ depend on two momenta squared and the nucleon mass: $s, q^{2}$ and $k^{2}=M_{N}^{2}$. Orbital momentum expansion functions, $X_{\alpha_{1} \ldots \alpha_{n}}^{(n)}\left(k_{\perp}\right)$ are presented in Appendix A. Polarization vectors of gamma obey the constraint for vector particles: $\epsilon_{\mu} q_{\mu}=0$.

\subsubsection{The (-)-vertices}

Vertices of the (-)-states $\left(J^{P}=\frac{1}{2}^{+}, \frac{3}{2}^{-}, \frac{5}{2}^{+}, \ldots\right)$ read:

$$
\begin{aligned}
& G_{\alpha_{1} \ldots \alpha_{n}}^{(a-)}\left(k_{\perp}\right)=G_{n+\frac{1}{2}}^{(a-)}\left(s, q^{2}\right) V_{\alpha_{1} \ldots \alpha_{n}}^{(a-) \mu}\left(k_{\perp}\right) \epsilon_{\mu}^{\perp q}, \quad a=1,2,3 \\
& V_{\alpha_{1} \ldots \alpha_{n}}^{(1-) \mu}\left(k_{\perp}\right)=\gamma_{\nu}^{\perp p} \gamma_{\mu} X_{\nu \alpha_{1} \ldots \alpha_{n}}^{(n+1)}\left(k_{\perp}\right), \\
& V_{\alpha_{1} \ldots \alpha_{n}}^{(2-) \mu}\left(k_{\perp}\right)=X_{\mu \alpha_{1} \ldots \alpha_{n}}^{(n+1)}\left(k_{\perp}\right), \\
& V_{\alpha_{1} \ldots \alpha_{n}}^{(3-) \mu}\left(k_{\perp}\right)=X_{\alpha_{2} \ldots \alpha_{L}}^{(n-1)}\left(k_{\perp}\right) g_{\alpha_{1} \mu}^{\perp p}
\end{aligned}
$$

\subsection{Operators at $q^{2} \neq 0$}

Equations (11), (3) are written for the convolution of operators with photon polarization vectors. To activate the photon polarization indices at $q^{2} \neq 0$ one can use 
the completeness conditions for polarization vectors which are written as follows:

$$
\sum_{a} \epsilon_{\mu^{\prime}}^{\perp q}(a) \epsilon_{\mu}^{\perp q *}(a)=g_{\mu^{\prime} \mu}-\frac{q_{\mu^{\prime}} q_{\mu}}{q^{2}}
$$

Therefore we write for operators at $q^{2} \neq 0$ :

$$
G_{\alpha_{1} \ldots \alpha_{n}}^{(a \pm) \mu}\left(k_{\perp}\right)=G_{n+\frac{1}{2}}^{(a \pm)}\left(s, q^{2}\right) V_{\alpha_{1} \ldots \alpha_{n}}^{(a \pm) \mu^{\prime}}\left(k_{\perp}\right)\left(g_{\mu^{\prime} \mu}-\frac{q_{\mu^{\prime}} q_{\mu}}{q^{2}}\right), \quad a=1,2,3
$$

The operators (5) are appropriate for working at $q^{2} \neq 0$, for example, with massive vector particles, $\left(q^{2}=M^{2}\right)$.

The pole singularity $\left(1 / q^{2}\right)$ can be eliminated, if it is necessary, by the form factor constraint: $G_{n+\frac{1}{2}}^{(a \pm)}\left(s, q^{2}=0\right)=0$. But in some states it turns out to amusing results. Another way to eliminate the $\left(1 / q^{2}\right)$-pole is related to the introduction of the longitudinal component.

\section{Longitudinal component and generalized Siegert theorem}

The elimination of the pole singularity can be performed by adding to the opera-

tor $\left(g_{\mu^{\prime} \mu}-\frac{q_{\mu^{\prime}} q_{\mu}}{q^{2}}\right)$ a longitudinal component (orthogonal to $q$ ) with an appropriate constant $A$ :

$$
\begin{aligned}
& \left(g_{\mu^{\prime} \mu}-\frac{q_{\mu^{\prime}} q_{\mu}}{q^{2}}\right) \rightarrow\left[g_{\mu^{\prime} \mu}-\frac{q_{\mu^{\prime}} q_{\mu}}{q^{2}}+A q_{\mu^{\prime}}\left(q_{\mu}-\frac{q^{2}}{(p q)} p_{\mu}\right)\right]_{A=1 / q^{2}} \\
& =\left[g_{\mu^{\prime} \mu}-\frac{q_{\mu^{\prime}} q_{\mu}}{q^{2}}+\frac{1}{q^{2}} q_{\mu^{\prime}}\left(q_{\mu}-\frac{q^{2}}{(p q)} p_{\mu}\right)\right]=\left(g_{\mu^{\prime} \mu}-\frac{q_{\mu^{\prime}} p_{\mu}}{(p q)}\right)
\end{aligned}
$$

The singularity $1 / q^{2}$ is cancelled but we face a new singularity at $(p q)=0$, this one can be eliminated by a zero form factor. But the particularity consists in the fact that the $1 /(p q)$ pole is related to the longitudinal component, therefore one can construct two transverse operators without the $1 /(p q)$ pole singularities.

Correspondingly, one vertex form factor should satisfy the generalized Siegert theorem, we nominate this operator (and form factor) as $a=3$ :

$$
\left[G_{J}^{(3 \pm)}\left(s, q^{2}\right)\right]_{s=M_{N}^{2}-q^{2}}=0, \quad a=3 .
$$

Also, let us emphasize that the longitudinal operator $L_{\mu}=\left(q_{\mu}-\frac{q^{2}}{(p q)} p_{\mu}\right)$ which is attended at (6) is nilpotent at $q^{2}=0:\left(L_{\mu} L_{\mu}=0\right)$. The nilpotent operators were used in [18] for the description of photon interactions with mesons.

\subsection{Separation of the longitudinal operators and analyticity constraint}

Separation of the longitudinal operators is performed here in detail. 


\subsubsection{The operators for the (-)-states}

To perform the separation of the longitudinal and transverse operators let us fix the $V_{\alpha_{1} \ldots \alpha_{n}}^{G(3-) \mu}\left(k_{\perp}\right)$ as a basic one:

$$
\begin{aligned}
V_{\alpha_{1} \ldots \alpha_{n}}^{G(3-) \mu}\left(k_{\perp}\right) \equiv \mathfrak{V}_{\alpha_{1} \ldots \alpha_{n}}^{(3-) \mu}\left(k_{\perp}\right) & =X_{\alpha_{2} \ldots \alpha_{n}}^{(n-1)}\left(k_{\perp}\right) g_{\alpha_{1} \mu^{\prime}}^{\perp p}\left(g_{\mu^{\prime} \mu}-\frac{q_{\mu^{\prime}} p_{\mu}}{(p q)}\right) \\
& =X_{\alpha_{2} \ldots \alpha_{n}}^{(n-1)}\left(k_{\perp}\right)\left(g_{\alpha_{1} \mu}-\frac{q_{\alpha_{1}} p_{\mu}}{(p q)}\right)
\end{aligned}
$$

The pole singularity at $(p q)=0$ should be eliminated by requirement (7), that is the generalized Siegert theorem.

Let us now consider the vertex $V_{\alpha_{1} \ldots \alpha_{n}}^{G(2-) \mu}\left(k_{\perp}\right)$ :

$$
\begin{aligned}
V_{\alpha_{1} \ldots \alpha_{n}}^{G(2-) \mu}\left(k_{\perp}\right) & =X_{\mu^{\prime} \alpha_{1} \ldots \alpha_{n}}^{(n+1)}\left(k_{\perp}\right)\left(g_{\mu^{\prime} \mu}-\frac{q_{\mu^{\prime}} p_{\mu}}{(p q)}\right) \\
& =\left(X_{\mu \alpha_{1} \ldots \alpha_{n}}^{(n+1)}\left(k_{\perp}\right)+X_{\mu^{\prime} \alpha_{1} \ldots \alpha_{n}}^{(n+1)}\left(k_{\perp}\right) \frac{k_{\mu^{\prime}}^{\perp} p_{\mu}}{(p q)}\right) \\
& =\left(X_{\mu \alpha_{1} \ldots \alpha_{n}}^{(n+1)}\left(k_{\perp}\right)-k_{\perp}^{2} \frac{2 n-1}{n} X_{\alpha_{2} \ldots \alpha_{n}}^{(n-1)}\left(k_{\perp}\right) g_{\alpha_{1} \mu}\right. \\
& \left.+k_{\perp}^{2} \frac{2 n-1}{n} X_{\alpha_{2} \ldots \alpha_{n}}^{(n-1)}\left(k_{\perp}\right)\left(g_{\alpha_{1} \mu}-\frac{q_{\alpha_{1}} p_{\mu}}{(p q)}\right)\right) .
\end{aligned}
$$

The last term is proportional to $V_{\alpha_{1} \ldots \alpha_{n}}^{G(3-) \mu}\left(k_{\perp}\right)$ and can be removed. Recall $k_{\perp}=$ $-q+p \frac{(p q)}{p^{2}}$ and $k_{\perp}^{2}=q^{2}-\frac{(p q)^{2}}{p^{2}}$. Also, we have used:

$$
X_{\mu^{\prime} \alpha_{1} \ldots \alpha_{n}}^{(n+1)}\left(k_{\perp}\right) \frac{k_{\mu^{\prime}}^{\perp} p_{\mu}}{(p q)}=k_{\perp}^{2} \frac{2 n-1}{n} X_{\alpha_{2} \ldots \alpha_{n}}^{(n-1)}\left(k_{\perp}\right)\left(-\frac{q_{\alpha_{1}} p_{\mu}}{(p q)}\right) .
$$

We see that the singular term can be removed by redefinition of the vertex:

$$
\begin{aligned}
V_{\alpha_{1} \ldots \alpha_{n}}^{G(2-) \mu}\left(k_{\perp}\right) & \rightarrow \mathfrak{V}_{\alpha_{1} \ldots \alpha_{n}}^{(2-) \mu}\left(k_{\perp}\right) \\
& =V_{\alpha_{1} \ldots \alpha_{n}}^{G(2-) \mu}\left(k_{\perp}\right)-q^{2} \frac{2 n-1}{n} V_{\alpha_{1} \ldots \alpha_{n}}^{G(3-) \mu}\left(k_{\perp}\right)
\end{aligned}
$$

The redefined vertex $\mathfrak{V}_{\alpha_{1} \ldots \alpha_{n}}^{(2-) \mu}\left(k_{\perp}\right)$ is analytical at $(p q)=0$ and $q_{\mu} \mathfrak{V}_{\alpha_{1} \ldots \alpha_{n}}^{(2-) \mu}\left(k_{\perp}\right)=0$.

Redefinition of the $V_{\alpha_{1} \ldots \alpha_{n}}^{G(1-) \mu}\left(k_{\perp}\right)$ looks as follows:

$$
\begin{aligned}
V_{\alpha_{1} \ldots \alpha_{n}}^{G(1-) \mu}\left(k_{\perp}\right) & =\gamma_{\nu}^{\perp p} \gamma_{\mu} X_{\nu \alpha_{1} \ldots \alpha_{n}}^{(n+1)}\left(k_{\perp}\right)-\gamma_{\nu}^{\perp p} \hat{q} X_{\nu \alpha_{1} \ldots \alpha_{n}}^{(n+1)}\left(k_{\perp}\right) \frac{p_{\mu}}{(p q)} \\
& =\gamma_{\nu}^{\perp p} \gamma_{\mu} X_{\nu \alpha_{1} \ldots \alpha_{n}}^{(n+1)}\left(k_{\perp}\right)-\gamma_{\nu}^{\perp p} \hat{q} k_{\nu}^{\perp} \frac{2 n+1}{n+1} X_{\alpha_{1} \ldots \alpha_{n}}^{(n)}\left(k_{\perp}\right) \frac{p_{\mu}}{(p q)} \\
& =\gamma_{\nu}^{\perp p} \gamma_{\mu} X_{\nu \alpha_{1} \ldots \alpha_{n}}^{(n+1)}\left(k_{\perp}\right)-\hat{k}_{\perp} \hat{q} \frac{2 n+1}{n+1} \cdot \frac{2 n-1}{n} k_{\alpha_{1}}^{\perp} X_{\alpha_{2} \ldots \alpha_{n}}^{(n)}\left(k_{\perp}\right) \frac{p_{\mu}}{(p q)}
\end{aligned}
$$

and

$$
\hat{k}_{\perp} \hat{q}=(p q)-\frac{(p q)}{p^{2}} \hat{p} \hat{k}-q^{2}
$$


To cancel the pole item in (12), $\sim 1 /(p q)$, we add $C \mathfrak{V}_{\alpha_{1} \ldots \alpha_{n}}^{(3-) \mu}\left(k_{\perp}\right)$ with proper coefficient $C$ :

$$
C=-q^{2} \frac{4 n^{2}-1}{n(n+1)}
$$

The redefined operator reads:

$$
\mathfrak{V}_{\alpha_{1} \ldots \alpha_{n}}^{(1-) \mu}\left(k_{\perp}\right)=V_{\alpha_{1} \ldots \alpha_{n}}^{G(1-) \mu}\left(k_{\perp}\right)-q^{2} \frac{4 n^{2}-1}{n(n+1)} V_{\alpha_{1} \ldots \alpha_{n}}^{G(3-) \mu}\left(k_{\perp}\right)
$$

\subsubsection{The operators for the $(+)$-states}

We have three operators:

$$
\begin{aligned}
& V_{\alpha_{1} \ldots \alpha_{n}}^{G(1+) \mu}\left(k_{\perp}\right)=i \gamma_{\mu^{\prime}}^{\perp p} \gamma_{5} X_{\alpha_{1} \ldots \alpha_{n}}^{(n)}\left(k_{\perp}\right)\left(g_{\mu^{\prime} \mu}-\frac{q_{\mu^{\prime}} p_{\mu}}{(p q)}\right), \\
& V_{\alpha_{1} \ldots \alpha_{n}}^{G(2+) \mu}\left(k_{\perp}\right)=i \gamma_{\nu}^{\perp p} \gamma_{5} X_{\mu^{\prime} \nu \alpha_{1} \ldots \alpha_{n}}^{(n+2)}\left(k_{\perp}\right)\left(g_{\mu^{\prime} \mu}-\frac{q_{\mu^{\prime}} p_{\mu}}{(p q)}\right), \\
& V_{\alpha_{1} \ldots \alpha_{n}}^{G(3+) \mu}\left(k_{\perp}\right)=i \gamma_{\nu}^{\perp p} \gamma_{5} X_{\nu \alpha_{1} \ldots \alpha_{n-1}}^{(n)}\left(k_{\perp}\right) g_{\alpha_{n} \mu^{\prime}}^{\perp p}\left(g_{\mu^{\prime} \mu}-\frac{q_{\mu^{\prime}} p_{\mu}}{(p q)}\right) .
\end{aligned}
$$

The reorganized operators $V_{\alpha_{1} \ldots \alpha_{n}}^{G(a+) \mu}\left(k_{\perp}\right) \rightarrow \mathfrak{V}_{\alpha_{1} \ldots \alpha_{n}}^{(a+){ }_{n}}\left(k_{\perp}\right)($ with $a=1,2,3)$ read:

$$
\begin{aligned}
& V_{\alpha_{1} \ldots \alpha_{n}}^{G(1+) \mu}\left(k_{\perp}\right) \rightarrow \mathfrak{V}_{\alpha_{1} \ldots \alpha_{n}}^{(1+) \mu}\left(k_{\perp}\right)=V_{\alpha_{1} \ldots \alpha_{n}}^{G(1+) \mu}\left(k_{\perp}\right)-V_{\alpha_{1} \ldots \alpha_{n}}^{G(3+) \mu}\left(k_{\perp}\right) \\
& V_{\alpha_{1} \ldots \alpha_{n}}^{G(2+) \mu}\left(k_{\perp}\right) \rightarrow \mathfrak{V}_{\alpha_{1} \ldots \alpha_{n}}^{(2+) \mu}\left(k_{\perp}\right)=V_{\alpha_{1} \ldots \alpha_{n}}^{G(2+) \mu}\left(k_{\perp}\right)-q^{2} \frac{(2 n+1) n}{(2 n-1)(n+1)} V_{\alpha_{1} \ldots \alpha_{n}}^{G(3+) \mu}\left(k_{\perp}\right) \\
& V_{\alpha_{1} \ldots \alpha_{n}}^{G(3+) \mu}\left(k_{\perp}\right) \equiv \mathfrak{V}_{\alpha_{1} \ldots \alpha_{n}}^{(3+) \mu}\left(k_{\perp}\right) \\
& =i \gamma_{\nu}^{\perp p} \gamma_{5}\left(X_{\nu \alpha_{2} \ldots \alpha_{n}}^{(n)}\left(k_{\perp}\right) g_{\alpha_{1} \mu}^{\perp p}-X_{\nu \alpha_{2} \ldots \alpha_{n}}^{(n)}\left(k_{\perp}\right) q_{\alpha_{1}} \frac{p_{\mu}}{(p q)}\right) .
\end{aligned}
$$

We see that all operators are subjects of the gauge requirement: $q_{\mu} \mathfrak{V}_{\alpha_{1} \ldots \alpha_{n}}^{(a \pm) \mu}\left(k_{\perp}\right)=0$, $a=1,2,3$.

\subsection{The generalized Siegert theorem}

Operators with taken into account form factors read as follows:

$$
\mathfrak{G}_{\alpha_{1} \ldots \alpha_{n}}^{(a \pm) \mu}\left(k_{\perp}\right)=G_{J}^{(a \pm)}\left(s, q^{2}\right) \mathfrak{V}_{\alpha_{1} \ldots \alpha_{n}}^{(a+) \mu}\left(k_{\perp}\right), \quad a=1,2,3 .
$$

The operators $\mathfrak{V}_{\alpha_{1} \ldots \alpha_{n}}^{(a+) \mu}\left(k_{\perp}\right)$ are constricted in such a way that the pole singularity $1 /(p q)$ attends at $(a=3)$-component only. Correspondingly form factors $G^{(3 \pm)}\left(s, q^{2}\right)$ obey to generalized Siegert theorem presented in eq.(7): $G^{(3 \pm)}\left(s, q^{2}\right) \sim$ $\left(s-M_{N}^{2}+q^{2}\right)$. 


\section{Different versions for construction of the $( \pm)$-operators}

In the previous Section we constructed $(+)$-operators $\left(J^{P}=\frac{1}{2}^{-}, \frac{3}{2}^{+}, \frac{5}{2}^{-}, \ldots\right)$ and (-)-operators $\left(J^{P}=\frac{1}{2}^{+}, \frac{3}{2}^{-}, \frac{5}{2}^{+}, \ldots\right)$ which obey analyticity and gauge invariance constraints that are $\mathfrak{G}_{\alpha_{1} \ldots \alpha_{n}}^{(a+) \mu}\left(k_{\perp}\right)$ and $\mathfrak{G}_{\alpha_{1} \ldots \alpha_{n}}^{(a-) \mu}\left(k_{\perp}\right)$. One can construct other sets of operators, $\widetilde{\mathfrak{G}}_{\alpha_{1} \ldots \alpha_{n}}^{(a+) \mu}\left(k_{\perp}\right)=\mathfrak{G}_{\alpha_{1} \ldots \alpha_{n}}^{(a-) \mu}\left(k_{\perp}\right) \cdot i \gamma_{5}$ and $\widetilde{\mathfrak{G}}_{\alpha_{1} \ldots \alpha_{n}}^{(a-) \mu}\left(k_{\perp}\right)=\mathfrak{G}_{\alpha_{1} \ldots \alpha_{n}}^{(a+) \mu}\left(k_{\perp}\right) \cdot i \gamma_{5}$. We have four equivalent definitions of the $( \pm)$-operators:

$$
\begin{array}{l|l}
\mathfrak{G}_{\alpha_{1} \ldots \alpha_{n}}^{(1+) \mu}\left(k_{\perp}\right) & \mathfrak{G}_{\alpha_{1} \ldots \alpha_{n}}^{(1-) \mu}\left(k_{\perp}\right) \\
\mathfrak{G}_{\alpha_{1} \ldots \ldots \alpha_{n}}^{(+) \mu}\left(k_{\perp}\right) & \mathfrak{G}_{\alpha_{1} \ldots \alpha_{n}}^{(2-) \mu}\left(k_{\perp}\right) \\
\mathfrak{G}_{\alpha_{1} \ldots \alpha_{n}}^{(3+) \mu}\left(k_{\perp}\right) & \mathfrak{G}_{\alpha_{1} \ldots \alpha_{n}}^{(3-) \mu}\left(k_{\perp}\right) \\
\hline \widetilde{\mathfrak{G}}_{\alpha_{1} \ldots \alpha_{n}}^{(1+) \mu}\left(k_{\perp}\right)=\mathfrak{G}_{\alpha_{1} \ldots \alpha_{n}}^{(1--) \mu}\left(k_{\perp}\right) \cdot i \gamma_{5} & \widetilde{\mathfrak{G}}_{\alpha_{1} \ldots \alpha_{n}}^{(1-) \mu}\left(k_{\perp}\right)=\mathfrak{G}_{\alpha_{1} \ldots \alpha_{n}}^{(1+) \mu}\left(k_{\perp}\right) \cdot i \gamma_{5} \\
\widetilde{\mathfrak{G}}_{\alpha_{1} \ldots \alpha_{n}}^{(2+) \mu}\left(k_{\perp}\right)=\mathfrak{G}_{\alpha_{1} \ldots \alpha_{n}}^{(2-) \mu}\left(k_{\perp}\right) \cdot i \gamma_{5} & \widetilde{\mathfrak{G}}_{\left.\alpha_{1} \ldots\right) \mu}^{(2-) \alpha_{n}}\left(k_{\perp}\right)=\mathfrak{G}_{\alpha_{1} \ldots \alpha_{n}}^{(2+) \mu}\left(k_{\perp}\right) \cdot i \gamma_{5} \\
\widetilde{G}_{\alpha_{1} \ldots \alpha_{n}}^{(3+) \mu}\left(k_{\perp}\right)=\mathfrak{G}_{\alpha_{1} \ldots \alpha_{n}}^{(3-) \mu}\left(k_{\perp}\right) \cdot i \gamma_{5} & \widetilde{\mathfrak{G}}_{\alpha_{1} \ldots \alpha_{n}}^{(3-) \mu}\left(k_{\perp}\right)=\mathfrak{G}_{\alpha_{1} \ldots \alpha_{n}}^{(3+) \mu}\left(k_{\perp}\right) \cdot i \gamma_{5}
\end{array}
$$

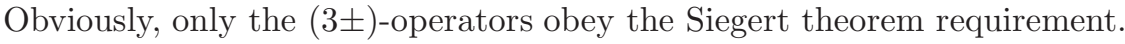

\section{Conclusion}

We construct gauge invariant operators for vertices of baryon resonances decaying into the nucleon-gamma state, $B\left(J^{P}\right) \rightarrow N\left(\frac{1}{2}^{+}\right)+\gamma$. The operators describe transition vertices of resonances with arbitrary spin-parity, $J^{P}$, and arbitrary momenta squared of gamma, $q^{2}$. The suggested operator construction presents the generalization of the scheme of ref. [17] for photon vertices, $\left(q^{2}=0\right)$.

The key point in the construction of the gauge invariant operators valid at all possible $q^{2}$ is the introduction of longitudinal spin operator $L_{\mu}$ which is nilpotent at $q^{2}=0:\left[L_{\mu} L_{\mu}\right]_{q^{2}=0}=0$. The addition of the nilpotent operator corrects the analytical structure of spin operators. For meson states such a correction was carried out in [18]20] (see also [21]22]). The procedure for baryon-gamma states is performed here (Section 3): the pole singularity inherent to spin operators of vector particles $\left(1 / q^{2}\right)$ is eliminated by adding the nilpotent operator $L_{\mu}$.

Form factors of the transitions $B\left(J^{P}\right) \rightarrow N\left(\frac{1}{2}^{+}\right)+\gamma$ should satisfy the generalized Siegert requirement: $G_{J}^{(3 \pm)}\left(s, q^{2}\right)=0$ at $s=M_{N}^{2}-q^{2}$.

\section{Acknowledgement}

We thank M.A. Matveev, V.A. Nikonov, J. Nyiri for useful discussions. The paper was supported by grant RSF 16-12-10267.

\section{Appendix A: Angular momentum operators for two-meson systems}

We use angular momentum operators $X_{\mu_{1} \ldots \mu_{L}}^{(L)}\left(k^{\perp}\right), Z_{\mu_{1} \ldots \mu_{L}}^{\alpha}\left(k^{\perp}\right)$ and the projection operator $O_{\nu_{1} \ldots \nu_{L}}^{\mu_{1} \ldots \mu_{L}}(\perp P)($ see $[18[21] 22])$. Let us recall their definition. 
The operators are constructed from the relative momenta $k_{\mu}^{\perp}$ and tensor $g_{\mu \nu}^{\perp}$. Both of them are orthogonal to the total momentum of the system:

$$
k_{\mu}^{\perp}=\frac{1}{2} g_{\mu \nu}^{\perp}\left(k_{1}-k_{2}\right)_{\nu}=k_{1 \nu} g_{\nu \mu}^{\perp P}=-k_{2 \nu} g_{\nu \mu}^{\perp P}, \quad g_{\mu \nu}^{\perp}=g_{\mu \nu}-\frac{P_{\mu} P_{\nu}}{s} .
$$

The operator for $L=0$ is a scalar (we write $X^{(0)}\left(k^{\perp}\right)=1$ ), and the operator for $L=1$ is a vector, $X_{\mu}^{(1)}=k_{\mu}^{\perp}$. The operators $X_{\mu_{1} \ldots \mu_{L}}^{(L)}$ for $L \geq 1$ can be written in the form of a recurrency relation:

$$
\begin{aligned}
& X_{\mu_{1} \ldots \mu_{L}}^{(L)}\left(k^{\perp}\right)=k_{\alpha}^{\perp} Z_{\mu_{1} \ldots \mu_{L}}^{\alpha}\left(k^{\perp}\right) \equiv k_{\alpha}^{\perp} Z_{\mu_{1} \ldots \mu_{L}, \alpha}\left(k^{\perp}\right), \\
& Z_{\mu_{1} \ldots \mu_{L}}^{\alpha}\left(k^{\perp}\right) \equiv Z_{\mu_{1} \ldots \mu_{L}, \alpha}\left(k^{\perp}\right)=\frac{2 L-1}{L^{2}}\left(\sum_{i=1}^{L} X_{\mu_{1} \ldots \mu_{i-1} \mu_{i+1} \ldots \mu_{L}}^{(L-1)}\left(k^{\perp}\right) g_{\mu_{i} \alpha}^{\perp}-\right. \\
&\left.-\frac{2}{2 L-1} \sum_{\substack{i, j=1 \\
i<j}}^{L} g_{\mu_{i} \mu_{j}}^{\perp} X_{\mu_{1} \ldots \mu_{i-1} \mu_{i+1} \ldots \mu_{j-1} \mu_{j+1} \ldots \mu_{L} \alpha}^{(L-1)}\left(k^{\perp}\right)\right) .
\end{aligned}
$$

We have a convolution equality $X_{\mu_{1} \ldots \mu_{L}}^{(L)}\left(k^{\perp}\right) k_{\mu_{L}}^{\perp}=k_{\perp}^{2} X_{\mu_{1} \ldots \mu_{L-1}}^{(L-1)}\left(k^{\perp}\right)$, with $k_{\perp}^{2} \equiv$ $k_{\mu}^{\perp} k_{\mu}^{\perp}$, and the tracelessness property of $X_{\mu \mu \mu_{3} \ldots \mu_{L}}^{(L)}=0$. On this basis, one can write down the normalization condition for orbital angular operators:

$$
\int \frac{d \Omega}{4 \pi} X_{\mu_{1} \ldots \mu_{L}}^{(L)}\left(k^{\perp}\right) X_{\mu_{1} \ldots \mu_{L}}^{(L)}\left(k^{\perp}\right)=\alpha_{L} k_{\perp}^{2 L}, \quad \alpha_{L}=\prod_{l=1}^{L} \frac{2 l-1}{l},
$$

where the integration is performed over spherical variables $\int d \Omega /(4 \pi)=1$.

Iterating Eq. (21), one obtains the following expression for the operator $X_{\mu_{1} \ldots \mu_{L}}^{(L)}$ at $L \geq 1$ :

$$
\begin{aligned}
& X_{\mu_{1} \ldots \mu_{L}}^{(L)}\left(k^{\perp}\right)=\alpha_{L}\left[k_{\mu_{1}}^{\perp} k_{\mu_{2}}^{\perp} k_{\mu_{3}}^{\perp} k_{\mu_{4}}^{\perp} \ldots k_{\mu_{L}}^{\perp}-\right. \\
& -\frac{k_{\perp}^{2}}{2 L-1}\left(g_{\mu_{1} \mu_{2}}^{\perp} k_{\mu_{3}}^{\perp} k_{\mu_{4}}^{\perp} \ldots k_{\mu_{L}}^{\perp}+g_{\mu_{1} \mu_{3}}^{\perp} k_{\mu_{2}}^{\perp} k_{\mu_{4}}^{\perp} \ldots k_{\mu_{L}}^{\perp}+\ldots\right)+ \\
& +\frac{k_{\perp}^{4}}{(2 L-1)(2 L-3)}\left(g_{\mu_{1} \mu_{2}}^{\perp} g_{\mu_{3} \mu_{4}}^{\perp} k_{\mu_{5}}^{\perp} k_{\mu_{6}}^{\perp} \ldots k_{\mu_{L}}^{\perp}\right. \\
& \left.\left.+g_{\mu_{1} \mu_{2}}^{\perp} g_{\mu_{3} \mu_{5}}^{\perp} k_{\mu_{4}}^{\perp} k_{\mu_{6}}^{\perp} \ldots k_{\mu_{L}}^{\perp}+\ldots\right)+\ldots\right] .
\end{aligned}
$$

For the projection operators, one has:

$$
\begin{aligned}
& O=1, \quad O_{\nu}^{\mu}(\perp P)=g_{\mu \nu}^{\perp}, \\
& O_{\nu_{1} \nu_{2}}^{\mu_{1} \mu_{2}}(\perp P)=\frac{1}{2}\left(g_{\mu_{1} \nu_{1}}^{\perp} g_{\mu_{2} \nu_{2}}^{\perp}+g_{\mu_{1} \nu_{2}}^{\perp} g_{\mu_{2} \nu_{1}}^{\perp}-\frac{2}{3} g_{\mu_{1} \mu_{2}}^{\perp} g_{\nu_{1} \nu_{2}}^{\perp}\right) .
\end{aligned}
$$


For higher states, the operator can be calculated using the recurrent expression:

$$
\begin{aligned}
& O_{\nu_{1} \ldots \nu_{L}}^{\mu_{1} \ldots \mu_{L}}(\perp P)=\frac{1}{L^{2}}\left(\sum_{i, j=1}^{L} g_{\mu_{i} \nu_{j}}^{\perp} O_{\nu_{1} \ldots \nu_{j-1} \nu_{j+1} \ldots \nu_{L}}^{\mu_{1} \ldots \mu_{i-1} \mu_{i+1} \ldots \mu_{L}}(\perp P)-\right. \\
& \left.-\frac{4}{(2 L-1)(2 L-3)} \times \sum_{\substack{i<j \\
k<m}}^{L} g_{\mu_{i} \mu_{j}}^{\perp} g_{\nu_{k} \nu_{m}}^{\perp} O_{\nu_{1} \ldots \nu_{k-1} \nu_{k+1} \ldots \nu_{m-1} \nu_{m+1} \ldots \nu_{L}}^{\mu_{1} \ldots \mu_{i-1} \mu_{i+1} \ldots \mu_{j-1} \mu_{j+1} \ldots \mu_{L}}(\perp P)\right) .
\end{aligned}
$$

The projection operators obey the relations:

$$
\begin{aligned}
O_{\nu_{1} \ldots \nu_{L}}^{\mu_{1} \ldots \mu_{L}}(\perp P) X_{\nu_{1} \ldots \nu_{L}}^{(L)}\left(k^{\perp}\right) & =X_{\mu_{1} \ldots \mu_{L}}^{(L)}\left(k^{\perp}\right) \\
O_{\nu_{1} \ldots \nu_{L}}^{\mu_{1} \ldots \mu_{L}}(\perp P) k_{\nu_{1}} k_{\nu_{2}} \ldots k_{\nu_{L}} & =\frac{1}{\alpha_{L}} X_{\mu_{1} \ldots \mu_{L}}^{(L)}\left(k^{\perp}\right) .
\end{aligned}
$$

Hence, the product of the two $X^{L}\left(k_{\perp}\right)$ operators results in the Legendre polynomials as follows:

$$
X_{\mu_{1} \ldots \mu_{L}}^{(L)}\left(k_{1}^{\perp}\right)(-1)^{L} O_{\nu_{1} \ldots \nu_{L}}^{\mu_{1} \ldots \mu_{L}}(\perp P) X_{\nu_{1} \ldots \nu_{L}}^{(L)}\left(k_{2}^{\perp}\right)=\alpha_{L}\left(\sqrt{-k_{1}^{\perp 2}} \sqrt{-k_{2}^{\perp 2}}\right)^{L} P_{L}(z),
$$

where $z \equiv\left(-k_{1 \nu}^{\perp} k_{2 \nu}^{\perp}\right) /\left(\sqrt{-k_{1}^{\perp 2}} \sqrt{-k_{2}^{\perp 2}}\right)$.

\section{Appendix B: Projection operators for baryon resonance states with arbitrary $J$.}

The wave function of a resonance state with spin $J=n+1 / 2$, momentum $p$ and the effective mass term $\sqrt{s}$ is given by a tensor four-spinor $\psi_{\mu_{1} \ldots \mu_{n}}$. It satisfies the constraints

$$
(\hat{p}-\sqrt{s}) \psi_{\mu_{1} \ldots \mu_{n}}=0, \quad p_{\mu_{i}} \psi_{\mu_{1} \ldots \mu_{n}}=0, \quad \gamma_{\mu_{i}} \psi_{\mu_{1} \ldots \mu_{n}}=0,
$$

and the symmetry properties

$$
\begin{aligned}
\psi_{\mu_{1} \ldots \mu_{i} \ldots \mu_{j} \ldots \mu_{n}} & =\psi_{\mu_{1} \ldots \mu_{j} \ldots \mu_{i} \ldots \mu_{n}}, \\
g_{\mu_{i} \mu_{j}} \psi_{\mu_{1} \ldots \mu_{i} \ldots \mu_{j} \ldots \mu_{n}} & =g_{\mu_{i} \mu_{j}}^{\perp p} \psi_{\mu_{1} \ldots \mu_{i} \ldots \mu_{j} \ldots \mu_{n}}=0 .
\end{aligned}
$$

Conditions (28), (29) define the structure of the denominator of the fermion propagator (the projection operator) which can be written in the following form:

$$
\begin{aligned}
& F_{\nu_{1} \ldots \nu_{n}}^{\mu_{1} \ldots \mu_{n}}(p)=(-1)^{n}(\hat{p}+\sqrt{s}) \Phi_{\nu_{1} \ldots \nu_{n}}^{\mu_{1} \ldots \mu_{n}}(\perp p), \\
& \mathfrak{P}_{\nu_{1} \ldots \nu_{n}}^{\mu_{1} \ldots \mu_{n}}(p)=\frac{F_{\nu_{1} \ldots \nu_{n}}^{\mu_{1} \ldots \mu_{n}}(p)}{m^{2}-s-i m \Gamma(s)}
\end{aligned}
$$

The operator $\Phi_{\nu_{1} \ldots \nu_{n}}^{\mu_{1} \ldots \mu_{n}}(\perp p)$ describes the tensor structure of the propagator. It is equal to 1 for a $(J=1 / 2)$-particle and is proportional to $g_{\mu \nu}^{\perp p}-\gamma_{\mu}^{\perp} \gamma_{\nu}^{\perp} / 3$ for a particle with spin $J=3 / 2$ (remind that $\gamma_{\mu}^{\perp}=g_{\mu \nu}^{\perp p} \gamma_{\nu}$ ).

The conditions (29) are identical for fermion and boson projection operators and therefore the fermion projection operator can be written as:

$$
\Phi_{\nu_{1} \ldots \nu_{n}}^{\mu_{1} \ldots \mu_{n}}(\perp p)=O_{\alpha_{1} \ldots \alpha_{n}}^{\mu_{1} \ldots \mu_{n}}(\perp p) \phi_{\beta_{1} \ldots \beta_{n}}^{\alpha_{1} \ldots \alpha_{n}}(\perp p) O_{\nu_{1} \ldots \nu_{n}}^{\beta_{1} \ldots \beta_{n}}(\perp p) .
$$


The operator $\phi_{\beta_{1} \ldots \beta_{n}}^{\alpha_{1} \ldots \alpha_{n}}(\perp p)$ can be expressed in a rather simple form since all symmetry and orthogonality conditions are imposed by $O$-operators. First, the $\phi$ operator is constructed of metric tensors only, which act in the space of $\perp p$ and $\gamma^{\perp}$-matrices. Second, a construction like $\gamma_{\alpha_{i}}^{\perp} \gamma_{\alpha_{j}}^{\perp}=\frac{1}{2} g_{\alpha_{i} \alpha_{j}}^{\perp}+\sigma_{\alpha_{i} \alpha_{j}}^{\perp}$ (remind that here $\sigma_{\alpha_{i} \alpha_{j}}^{\perp}=\frac{1}{2}\left(\gamma_{\alpha_{i}}^{\perp} \gamma_{\alpha_{j}}^{\perp}-\gamma_{\alpha_{j}}^{\perp} \gamma_{\alpha_{i}}^{\perp}\right)$ gives zero if multiplied by an $O_{\alpha_{1} \ldots \alpha_{n}}^{\mu_{1} \ldots \mu_{n}}$-operator: the first term is due to the traceless conditions and the second one to symmetry properties. The only structures which can then be constructed are $g_{\alpha_{i} \beta_{j}}^{\perp}$ and $\sigma_{\alpha_{i} \beta_{j}}^{\perp}$. Moreover, taking into account the symmetry properties of the $O$-operators, one can use any pair of indices from sets $\alpha_{1} \ldots \alpha_{n}$ and $\beta_{1} \ldots \beta_{n}$, for example, $\alpha_{i} \rightarrow \alpha_{1}$ and $\beta_{j} \rightarrow \beta_{1}$. Then

$$
\phi_{\beta_{1} \ldots \beta_{n}}^{\alpha_{1} \ldots \alpha_{n}}(\perp p)=\frac{n+1}{2 n+1}\left(g_{\alpha_{1} \beta_{1}}^{\perp}-\frac{n}{n+1} \sigma_{\alpha_{1} \beta_{1}}^{\perp}\right) \prod_{i=2}^{n} g_{\alpha_{i} \beta_{i}}^{\perp} .
$$

Since $\Phi_{\nu_{1} \ldots \nu_{n}}^{\mu_{1} \ldots \mu_{n}}(\perp p)$ is determined by convolutions of $O$-operators, see Eq. (31), we can replace in (31)

$$
\phi_{\beta_{1} \ldots \beta_{n}}^{\alpha_{1} \ldots \alpha_{n}}(\perp p) \rightarrow \phi_{\beta_{1} \ldots \beta_{n}}^{\alpha_{1} \ldots \alpha_{n}}(p)=\frac{n+1}{2 n+1}\left(g_{\alpha_{1} \beta_{1}}-\frac{n}{n+1} \sigma_{\alpha_{1} \beta_{1}}\right) \prod_{i=2}^{n} g_{\alpha_{i} \beta_{i}} .
$$

The coefficients in (33) are chosen to satisfy the constraints (28) and the convolution condition:

$$
F_{\alpha_{1} \ldots \alpha_{n}}^{\mu_{1} \ldots \mu_{n}}(p) F_{\nu_{1} \ldots \nu_{n}}^{\alpha_{1} \ldots \alpha_{n}}(p)=(-1)^{n} F_{\nu_{1} \ldots \nu_{n}}^{\mu_{1} \ldots \mu_{n}}(p) \cdot 2 \sqrt{s}
$$

The amplitude of photo-production (or, electro-production) of baryon resonances on nucleon target reads:

$$
\mathfrak{A}_{\mu_{1} \ldots \mu_{n}}(p)=\frac{F_{\alpha_{1} \ldots \alpha_{n}}^{\mu_{1} \ldots \mu_{n}}(p)}{m^{2}-s-i m \Gamma(s)} \mathfrak{G}_{\alpha_{1} \ldots \alpha_{n}}^{(a \pm)}\left(k_{\perp}\right)
$$

remind, $a=1,2,3$.

\section{References}

1. H.L. Anderson, E. Fermi, R. Martin, D.E. Nagle, Phys. Rev. 91, 155 (1953).

2. G.F. Chew, Phys. Rev. 95, 1669 (1954).

3. M. Gell-Mann, K.M. Watson, Ann. Rev. Nucl. Sci., Standford, 1954.

4. M. Gell-Mann, M.L. Goldberger, W.E. Thirring, Phys. Rev. 95, 1612 (1954).

5. M. Gell-Mann and M. L. Goldberger, Phys. Rev. 96, 1433 (1954).

6. N. M. Kroll and M. A. Ruderman, Phys. Rev. 93, 233 (1954).

7. R. C. E. Devenish, T. S. Eisenschitz and J. G. Keorner, Phys. Rev. D 14, 3063 (1976).

8. I. Denisenko et al. Phys.Lett. B755 (2016) 97.

9. A. Wilson et al. (CBELSA/TAPS Collaboration) Phys.Lett. B749 (2015) 407.

10. V. Sokhoyan et al. (CBELSA/TAPS Collaboration ), Eur.Phys.J. A51 (2015) 95.

11. A.V. Anisovich et al., J.Phys. G37 (2010) 025004.

12. A.V. Anisovich et al., Eur.Phys.J. A41 (2009) 13-24.

13. I.G. Aznauryan et al. (CLAS Collaboration), Phys.Rev. C80 (2009) 055203.

14. N. Sparks et al. (CBELSA/TAPS Collaboration), Phys.Rev. C81 (2010) 065210.

15. A. Wilson et al. (CBELSA/TAPS Collaboration ), Phys.Lett. B749 (2015) 407-413. 
16. I. Horn et al. (CB-ELSA Collaboration), Eur.Phys.J. A38 (2008) 173-186 .

17. A.V. Anisovich, A.V. Sarantsev, Partial decay widths of baryons in the spin-momentum operator expansion method, Eur.Phys.J. A30 (2006) 427-441, hep-ph/0605135.

18. A.V. Anisovich, V.V. Anisovich, V.N. Markov, M.A. Matveev, V.A. Nikonov, A.V. Sarantsev, Radiative decays of quarkonium states, momentum operator expansion and nilpotent operators, J.Phys. G31 (2005) 1537, hep-ph/0509042.

19. A.V. Anisovich, A. Sarantsev, O. Bartholomy, E. Klempt, V.A. Nikonov, U. Thoma, Photoproduction of baryons decaying into $N \pi$ and $N \eta$, Eur.Phys.J. A25 (2005) 427, hep-ex/0506010.

20. V.V. Anisovich, M.A. Matveev, Description of composite systems in the spectral integration technique: The gauge invariance and analyticity constraints for the radiative decay amplitudes , Phys.Atom.Nucl. 67 (2004) 614, [ Yad.Fiz. 67 (2004) 634], hep-ph/0303119.

21. A.V. Anisovich, V.V. Anisovich, M.A. Matveev, V.A. Nikonov, J. Nyiri, A.V. Sarantsev, Mesons and baryons. Systematization and methods of analisis., World Scientific Publishing (2008).

22. A.V. Anisovich, V.V. Anisovich, M.A. Matveev, V.A. Nikonov, J. Nyiri, A.V. Sarantsev, Three-particle physics and dispersion relation theory, World Scientific Publishing (2013).

23. A.J.F. Siegert, Phys. Rev. 52 (1937) 317 